\title{
Orchids of Mount Ungaran (Indonesia) compiled from a decade of data collections between 2010 and 2021
}

\author{
FIRMAN HERU KURNIAWAN ${ }^{1, \vartheta}$, LUTFIAN NAZAR ${ }^{1}$, RINI ANJARWATI $^{2}$, HERBOWO DWI SASONO ${ }^{2}$, \\ MARGARETA RAHAYUNINGSIH ${ }^{2}$ \\ ${ }^{1}$ Yayasan Akar Banir Indonesia. Jl. Durian 3, No. 63, Semarang 50229, Central Java, Indonesia. `email: anggrekungaran@gmail.com \\ ${ }^{2}$ Department of Biology, Faculty of Mathematics and Natural Sciences, Universitas Negeri Semarang. Jl. Raya Sekaran, Semarang 50299, Central Java, \\ Indonesia
}

Manuscript received: 28 September 2021. Revision accepted: 30 November 2021.

\begin{abstract}
Kurniawan FH, Nazar L, Anjarwati R, Sasono HD, Rahayuningsih M. 2021. Orchids of Mount Ungaran (Indonesia) were compiled from a decade of data collection between 2010 and 2021. Nusantara Bioscience 13: 238-252. Orchid is well known as a plant group widely used as ornamental plants because of their uniqueness. But, high exploitation, deforestation, and land-use changes have threatened orchid species and triggered extinction. It also happened in Mount Ungaran, Central Java, Indonesia, despite its designation as an essential ecosystem area. This research was conducted to add an updated list of wild orchid species that can serve as biodiversity baseline data for conservation management in Mount Ungaran. The study was carried out through exploration activities from 2010 to 2021. The study area covered 11 sites, i.e., Gentong, Kalisidi, Pasigitan, Banyuwindu, Watuondo, Indrakila, Gonoharjo, Gajahmungkur, Camp Mawar, Gondang, and Puncak, which were all included in the buffer zone of Mount Ungaran. A total of 115 species from 54 genera of orchids were successfully identified, including 78 species of epiphyte and 37 species of terrestrial orchids. Among the species recorded, at least 27 species were known to be endemic to Indonesia, and 8 species were endemic to the island of Java, of which four species were listed in the IUCN Red List. In addition, all the species found were listed in Appendix II of the CITES, except one species, Paphiopedilum javanicum, which was listed in Appendix I. This study recorded the new distribution records of several species of orchids that were previously only found in West Java or East Java, i.e., Ania penangiana, Pholidota convallariae, and Crepidium junghuhnii. This study has also documented a species from the genus Crepidium, which morphological characters have not been described in any identification guides. The Gentong area has the potential as a priority for in-situ orchid protection area of the highest number of species reached $54 \%$ of all orchids found on Mount Ungaran.
\end{abstract}

Keywords: Diversity, Mount Ungaran, Orchidaceae, wild orchid

\section{INTRODUCTION}

The Orchidaceae (orchid group) is one of the largest families in the plant kingdom, consisting of about 28,000 currently accepted species and some 800 subspecies distributed in about 763 genera (Biswas and Singh 2019). The Orchidaceae family can be divided into five subfamilies: Apostasioideae, Cypripedioideae, Epidendroideae, Orchidoideae, and Vanilloideae (Chase et al. 2015; Dong et al. 2018). As a mega-biodiversity country in the tropical equator, Indonesia consists of at least 5,000 species of orchids (Hariyanto et al. 2020). The presence of mountains in Java contributed to the high value of wild orchids. It is recognized that Java has at least 731 species within 130 genera (Comber 1990). For example, Mount Ungaran in Central Java, with an extent of 5,500 ha, comprises forested areas and mixed plantations that serve as habitats for various species, including wild orchids.

Because of the richness of their members, orchids have a variety of diverse growth forms, such as lithophytes, terrestrials, epiphytes, to saprophytes. It's the same with leaf shapes ranging from pencil form, elongated, rounded, peltate, etc. Like most monocotyledonous plants, the orchid lamina exhibits parallel leaf veins types with thicknesses that vary from thin to fleshy. Orchid species may be recognized by the unique flower structures, which show three sepals, one on the back called the dorsal sepals, while the other two are called lateral sepals. In addition, orchids also have two lateral petals and one differentiation petal (Artaka 2019; De 2020). This differentiation petal is modified as labellum and column, the modification to selfpollination and even attract their pollinators (Attri and Kant 2011).

Orchids have ecological, economic, and even health benefits. In ecology, orchids are bio-indicators of environmental health due to their sensitivity to environmental changes, play a role in the nitrogen cycle, and support soil composition due to their association with mycorrhizae (Seaton et al. 2013; Nurfadilah et al. 2016). Economically, orchids are popular as ornamental plants; thus, wild orchids have the potential as a parental lineage in a breeding program to produce beautiful desired varieties (Sadili and Sundari 2017). Regarding health benefits, some orchid species are herbal medicines such as pain relievers, anti-inflammatory, anti-rheumatic, and digestive, and even used as toners and beauty soaps (Asseleih et al. 2015). Unfortunately, such uses often reduce the natural population of some orchid species and even cause local extinction. 
Despite being designated as an important bird and biodiversity area (Birdlife International 2021), some forested areas in Mount Ungaran have been deforested (up to $31.5-40.1 \%$ ) during the $2000-2005$ periods due to agricultural expansion and settlement development (Rahayuningsih et al. 2015; Rahayuningsih et al. 2020). Furthermore, other data showed that from 1991-2009, about $75 \%$ of the forest of Mount Ungaran was converted to other land uses (Rahayuningsih et al. 2017). In addition, the existence of orchids in Mount Ungaran is also threatened by illegal collection by orchid hunters. Altogether, these threats put pressure on various types of orchids to endangerment, moreover for species that have not been recorded (Agustini et al. 2016; Besi et al. 2019).

Several studies regarding the conservation of Mount Ungaran have been carried out, including data collection on birds and another biodiversity (Rahayuningsih et al. 2015; Rahayuningsih et al. 2017; Rahayuningsih et al. 2020). However, the study on orchids of Mount Ungaran has not been fully revealed, while the threats to the plant, as mentioned above, continue unabated. Over many years, wild orchids' botanical collections have been focused on ex-situ species conservation to save them from extinction (Besi et al. 2019). Although a book on orchids in Ungaran has been published for a conservation purposes (Utami et al. 2018), some anecdotal information about undocumented species has not yet been published scientifically.
Therefore, this study aimed to inventory orchids in a broader scope of Mount Ungaran to provide an updated list of orchid species in the area. The documented species will be accompanied by data on species composition based on the area studied, life types, current global distribution, and their conservation status. The result of this study can be used as baseline information to support the ongoing conservation and management program, including the plan for establishing Mount Ungaran as an Essential Ecosystem Area (Kawasan Ekosistem Esensial/KEE).

\section{MATERIALS AND METHODS}

\section{Study sites}

Mount Ungaran is located in Central Java Province, Indonesia (S $7^{\circ} 12^{\prime}$ E $\left.110^{\circ} 20^{\prime}\right)$, one of the remaining forest areas on Java Island that Perhutani manages. Mount Ungaran has 5,500 ha, covered by two regencies, i.e., Kendal and Semarang provinces of Central Java, Indonesia. Mount Ungaran has an average temperature range from 22 to $27^{\circ} \mathrm{C}$ and an altitude range between $900-2,050$ masl. Mount Ungaran has several habitat types, i.e., primary dry forest and secondary dry forest with coffee plantation under the canopy. The study took place in 11 sites, i.e., Gentong, Kalisidi, Pasigitan, Banyuwindu, Watuondo, Indrakila, Gonoharjo, Gajahmungkur, Camp Mawar, Gondang, and Puncak (Figure 1).

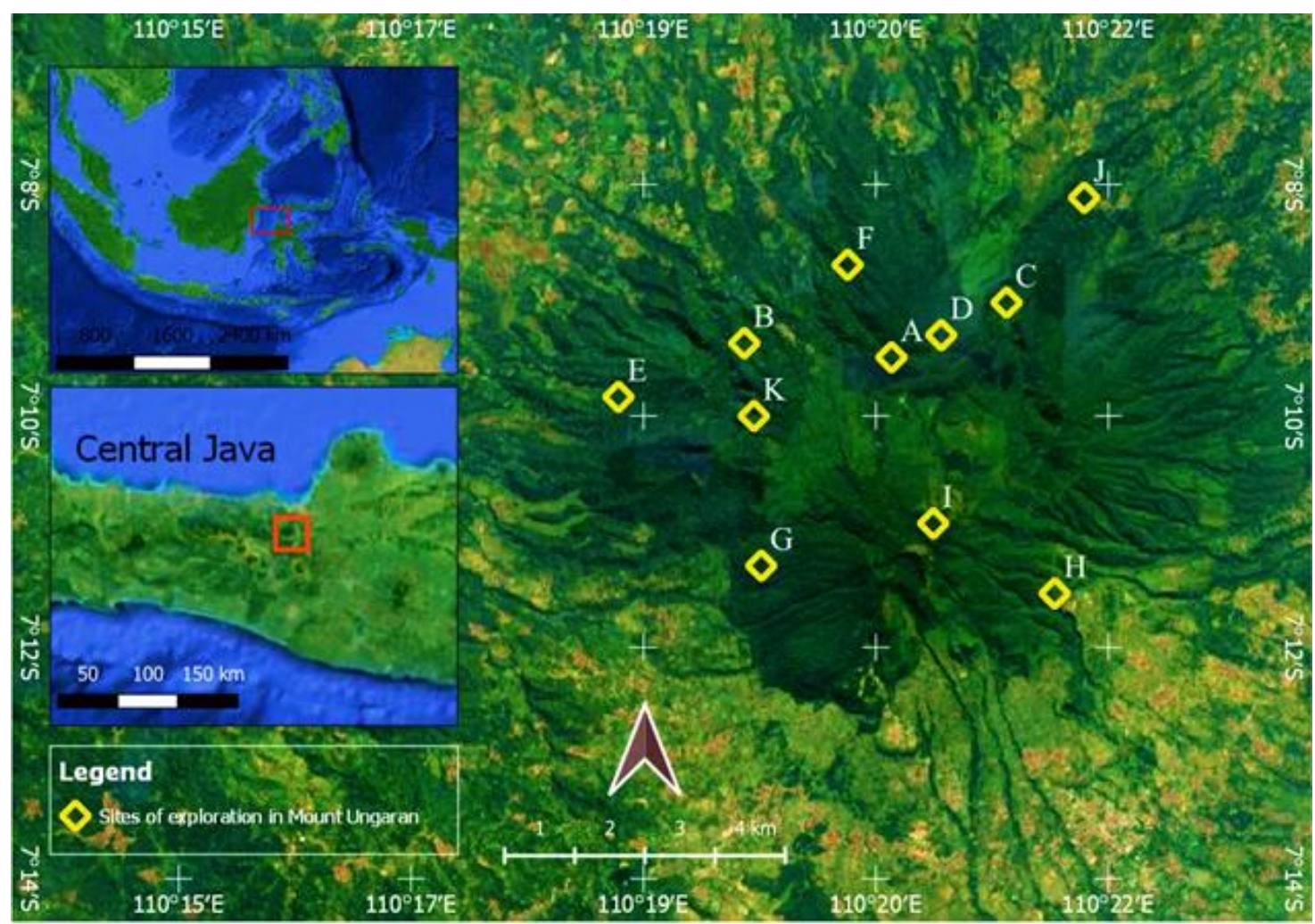

Figure 1. Map of the study sites of orchids exploration in Mount Ungaran, Central Java, Indonesia: A. Gentong, B. Watuondo, C. Kalisidi, D. Pasigitan, E. Banyuwindu, F. Gonoharjo, G. Gondang, H. Mawar Camp, I. Puncak, J. Indrakila (Source: Google Satelite and field data. Coordinates system WGS 84) 
Gentong, Kalisidi, Pasigitan, Banyuwindu, Watuondo, Gonoharjo, and Indrakila are sub-montane forests with altitudes between 900-1,500 m asl, represent the secondary forest with the domination of Syzygium sp., Weinmannia fraxinea and some of Lauraceae and Euphorbiaceae families. Under the canopy in, these areas are commonly used by localities as coffee plantations. Then, Gajahmungkur, Gondang, and Puncak are montane forests with ranges of altitudes 1,500-2,050 masl, represent the primary forest, and are relatively dominated by members of Fagaceae family (Lithocarpus sp., and Castanopsis sp.) (Figure 1). In addition, we explored Mawar Camp (1,0001,800 masl), a unique area with three vegetation types: homogenous pine plantation, primary forest, and intensive coffee plantation. Mawar Camp is used by localities as a legal tracking route to submit the mountain. We explored all of these areas from 2010 to 2021, divided into 3 periods: the first in 2010-2014, the second in 2014-2018, and the last third in 2018-2021.

\section{Data collection procedures}

Data were collected using an opportunistic sampling method by searching for species in habitats along defined pathways in every 11 sites. We used the photographic approach to minimize the collection of living specimens and the herbarium. The picture of each orchid organ in each species was as detailed as possible, including the habitus, leaves, stem, inflorescence, and flower. In addition, clear flower photographs must show that the petals, sepals, labellum, and column were conducted as important orchid characters for identification. Finally, GPS tagged each orchid species found in the pathways as a base for weekly monitoring to check the flowering periods.

\section{Data analyze}

The taxonomical identification used several sources, including Orchids of Java (Comber 1990), Die Orchideen von Java Figuren atlas (Smith 1908), and the online data from Swiss Orchids Foundation (https://orchid.unibas.ch/index.php/en/) The valid names and the current distribution data were retrieved based on the current data of The World Checklist of Selected Plant Families (Govaerts et al. 2021). Expert consultations were also sought to make an accurate identification. The protection status of each species was based on IUCN (http://www.iucnredlist.org), Index of CITES species (https://checklist.cites.org/\#/en), and Indonesian Regulation of Peraturan Menteri Lingkungan Hidup dan Kehutanan Republik Indonesia No. P.106/MenLHK/Setjen/Kum.1/ 12/2018 for validation (KLHK 2018).

\section{RESULTS AND DISCUSSION}

\section{Species composition}

Across the 11 sites surveyed in Mount Ungaran, a total of 115 orchid species were found, including 78 species that were epiphyte, and the remaining 37 species were terrestrial in their life type (Table 1). All the species found were successfully identified except one from the genus Crepidium. Among the 11 areas surveyed, Gentong had the highest number of species, with 61 species, while Indrakila had the lowest number, with only six species (Figure 2A). The second survey period found the most significant number of orchids, with a record of 93 identified species, followed by the first with 82 species and the third with 52 species (Figure 2B). We found 27 species endemic to Indonesia and eight endemics to Java island. The protection status of CITES includes 114 species in Appendix II, and only a species is in Appendix I, and National regulations did not protect all of these species. On the other hand, four species are protected by the IUCN Redlist, including Paphiopedilum javanicum (Reinw. ex Lindl.) Pfitzer, which is categorized as Endangered and Appendix I. 
Table 1. Orchids found and identified in Mount Ungaran, Central Java Province, Indonesia

\begin{tabular}{|c|c|c|c|c|c|c|}
\hline \multirow[b]{2}{*}{ Taxa } & \multirow[b]{2}{*}{$\begin{array}{l}\text { Growth } \\
\text { habit }\end{array}$} & \multicolumn{3}{|c|}{ Protection status } & \multirow[b]{2}{*}{ Global current distribution } & \multirow{2}{*}{$\begin{array}{l}\text { Specific } \\
\text { distribution in the } \\
\text { areas studied }\end{array}$} \\
\hline & & IUCN & CITES & $\begin{array}{c}\text { Indonesia } \\
\text { n law }\end{array}$ & & \\
\hline $\begin{array}{l}\text { Acriopsis liliifolia var. liliifolia } \\
\text { (J.Koenig) Ormerod }\end{array}$ & $\mathrm{E}$ & - & II & - & $\begin{array}{l}\text { Andaman Is., Bismarck Archipelago, Borneo, Cambodia, Caroline Is., East Himalaya, Jawa, } \\
\text { Laos, Lesser Sunda Is., Malaysia, Maluku, Myanmar, New Guinea, Philippines, Queensland, } \\
\text { Solomon Is., Sulawesi, Sumatera, Thailand, Vietnam }\end{array}$ & Ban, Maw, Gond \\
\hline Aerides odorata Lour. & $\mathrm{E}$ & - & II & - & $\begin{array}{l}\text { Andaman Is., Assam, Bangladesh, Borneo, Cambodia, China South-Central, China Southeast, } \\
\text { East Himalaya, India, Jawa, Laos, Lesser Sunda Is., Malaysia, Myanmar, Nepal, Nicobar Is., } \\
\text { Philippines, Sulawesi, Sumatera, Thailand, Vietnam, West Himalaya }\end{array}$ & Gen, Ban, Gond \\
\hline Agrostophyllum cyathiforme J.J.Sm. & $\mathrm{E}$ & $\mathrm{LC}$ & II & - & Borneo, Jawa, Malaysia, Sumatera & Maw, Gond \\
\hline $\begin{array}{l}\text { Agrostophyllum stipulatum subsp. } \\
\text { bicuspidatum (J.J.Sm.) Schuit. }\end{array}$ & $\mathrm{E}$ & - & II & - & Borneo, Jawa, Malaysia, Sulawesi, Sumatera & $\begin{array}{l}\text { Gen, Kal, Pas, } \\
\text { Gon, Ban, Gaj, } \\
\text { Wat, Gond }\end{array}$ \\
\hline $\begin{array}{l}\text { Ania penangiana (Hook.f.) } \\
\text { Summerh. }\end{array}$ & $\mathrm{T}$ & - & II & - & $\begin{array}{l}\text { Assam, Borneo, East Himalaya, Hainan, India, Jawa, Malaysia, Maluku, Nepal, New Guinea, } \\
\text { Sumatera, Taiwan, Thailand, Vietnam }\end{array}$ & Gen, Pas \\
\hline Anoectochilus reinwardtii Blume & $\mathrm{T}$ & - & II & - & Borneo, Jawa, Malaysia, Maluku, Sumatera, Thailand & Gen, Wat \\
\hline Appendicula alba Blume & $\mathrm{T}$ & - & II & - & Borneo, Jawa, Lesser Sunda Is., Malaysia, Philippines, Sulawesi, Sumatera, Thailand & Gen, Gaj, Gond \\
\hline Appendicula angustifolia Blume** & $\mathrm{E}$ & - & II & - & Jawa, Sumatera & $\begin{array}{l}\text { Gen, Kal, Pas, } \\
\text { Gon, Ban, Gaj, } \\
\text { Wat, Gond }\end{array}$ \\
\hline Appendicula ramosa Blume** & $\mathrm{E}$ & - & II & - & Jawa, Lesser Sunda Is., Sulawesi, Sumatera & Ban \\
\hline Appendicula reflexa Blume & $\mathrm{E}$ & - & II & - & $\begin{array}{l}\text { Bismarck Archipelago, Borneo, Cambodia, Caroline Is., Fiji, Jawa, Lesser Sunda Is., Malaysia, } \\
\text { Maluku, New Caledonia, New Guinea, Nicobar Is., Philippines, Santa Cruz Is., Solomon Is., } \\
\text { Sulawesi, Sumatera, Taiwan, Thailand, Tonga, Vanuatu, Vietnam, Wallis-Futuna Is. }\end{array}$ & Gen, Pun \\
\hline $\begin{array}{l}\text { Arundina graminifolia (D.Don) } \\
\text { Hochr. }\end{array}$ & $\mathrm{T}$ & - & II & - & $\begin{array}{l}\text { Assam, Bangladesh, Borneo, Cambodia, China South-Central, China Southeast, East Himalaya, } \\
\text { Hainan, India, Jawa, Laos, Lesser Sunda Is., Malaysia, Maluku, Myanmar, Nansei-shoto, Nepal, } \\
\text { Philippines, Sri Lanka, Sulawesi, Sumatera, Taiwan, Thailand, Tibet, Vietnam }\end{array}$ & Pun \\
\hline Bogoria raciborskii J.J.Sm.** & $\mathrm{E}$ & - & II & - & Borneo, Jawa & Ind \\
\hline $\begin{array}{l}\text { Bryobium retusum (Blume) Y.P.Ng } \\
\text { \& P.J.Cribb }\end{array}$ & $\mathrm{E}$ & - & II & - & Borneo, Christmas I., Jawa, Lesser Sunda Is., New Caledonia, Solomon Is., Sulawesi & Wat \\
\hline $\begin{array}{l}\text { Bulbophyllum angustifolium } \\
\text { (Blume) Lindl. }\end{array}$ & $\mathrm{E}$ & - & II & - & Jawa, Lesser Sunda Is., Malaysia, Sumatera & Gen, Gaj \\
\hline $\begin{array}{l}\text { Bulbophyllum biflorum Teijsm. \& } \\
\text { Binn. }\end{array}$ & $\mathrm{E}$ & - & II & - & Borneo, Jawa, Lesser Sunda Is., Malaysia, Sumatera, Thailand & $\begin{array}{l}\text { Gen, Kal, Pas, } \\
\text { Gon, Ban, Wat }\end{array}$ \\
\hline $\begin{array}{l}\text { Bulbophyllum capitatum (Blume) } \\
\text { Lindl.** }\end{array}$ & $\mathrm{E}$ & - & II & - & Borneo, Jawa & Gaj, Gaj, Gond \\
\hline $\begin{array}{l}\text { Bulbophyllum depressum King \& } \\
\text { Pantl }\end{array}$ & $\mathrm{E}$ & - & II & - & $\begin{array}{l}\text { Assam, Borneo, China South-Central, China Southeast, East Himalaya, Hainan, India, Jawa, } \\
\text { Laos, Lesser Sunda Is., Malaysia, Myanmar, Sumatera, Thailand, Vietnam }\end{array}$ & Gen, Pas, Wat \\
\hline $\begin{array}{l}\text { Bulbophyllum ecornutum subsp. } \\
\text { ecornutum (J.J.Sm.) J.J.Sm. }\end{array}$ & $\mathrm{E}$ & - & II & - & Borneo, Jawa, Lesser Sunda Is. & Wat \\
\hline Bulbophyllum flavidiflorum Carr** & E & - & II & - & Jawa, Lesser Sunda Is., Sumatera & Gaj, Gaj, Pun \\
\hline
\end{tabular}




\begin{tabular}{|c|c|c|c|c|c|c|}
\hline $\begin{array}{l}\text { Bulbophyllum gibbosum (Blume) } \\
\text { Lindl. }\end{array}$ & $\mathrm{E}$ & - & II & - & Borneo, Jawa, Lesser Sunda Is., Malaysia, Sumatera & Pun, Gond \\
\hline $\begin{array}{l}\text { Bulbophyllum laxiflorum (Blume) } \\
\text { Lindl. }\end{array}$ & $\mathrm{E}$ & - & II & - & $\begin{array}{l}\text { Borneo, Cambodia, Jawa, Laos, Malaysia, Myanmar, Philippines, Sulawesi, Sumatera, } \\
\text { Thailand, Vietnam }\end{array}$ & Gen \\
\hline $\begin{array}{l}\text { Bulbophyllum lobbii Lindl. subsp. } \\
\text { lobbii }\end{array}$ & $\mathrm{E}$ & - & II & - & $\begin{array}{l}\text { Assam, Bangladesh, Borneo, Cambodia, East Himalaya, Jawa, Lesser Sunda Is., Malaysia, } \\
\text { Myanmar, Philippines, Sumatera, Thailand }\end{array}$ & Gen, Kal, Ind \\
\hline Bulbophyllum stelis J.J.Sm**. & E & - & II & - & Jawa, Sumatera & Pun \\
\hline $\begin{array}{l}\text { Bulbophyllum sulcatum (Blume) } \\
\text { Lindl. }\end{array}$ & $\mathrm{E}$ & - & II & - & Jawa, Lesser Sunda Is., Malaysia, Sumatera, Thailand, Vietnam & Gaj, Gaj \\
\hline $\begin{array}{l}\text { Calanthe triplicata (Willemet) } \\
\text { Ames }\end{array}$ & $\mathrm{T}$ & - & II & - & $\begin{array}{l}\text { Andaman Is., Assam, Bismarck Archipelago, Borneo, Cambodia, Caroline Is., China South- } \\
\text { Central, China Southeast, East Himalaya, Fiji, Hainan, India, Jawa, Kazan-retto, Laos, Lesser } \\
\text { Sunda Is., Malaysia, Maluku, Marianas, Myanmar, Nansei-shoto, New Caledonia, New Guinea, } \\
\text { New South Wales, Norfolk Is., Ogasawara-shoto, Philippines, Queensland, Samoa, Society Is., } \\
\text { Solomon Is., Sri Lanka, Sulawesi, Sumatera, Taiwan, Thailand, Tonga, Tubuai Is., Vanuatu, } \\
\text { Vietnam, Wallis-Futuna Is. }\end{array}$ & $\begin{array}{l}\text { Gen, Kal, Pas, } \\
\text { Gon, Ban, Gaj, } \\
\text { Wat, Ind }\end{array}$ \\
\hline Ceratostylis backeri J.J.Sm.* & E & - & II & - & Jawa & Gaj, Pun \\
\hline Ceratostylis graminea Blume** & $\mathrm{E}$ & - & II & - & Jawa, Sumatera & Gen, Pas \\
\hline Ceratostylis radiata J.J.Sm. & $\mathrm{E}$ & - & II & - & Assam, Borneo, Jawa, Malaysia, Myanmar, Sumatera, Thailand, Tibet, Vietnam & Gen \\
\hline Ceratostylis subulata Blume & $\mathrm{E}$ & - & II & - & $\begin{array}{l}\text { Assam, Borneo, Cambodia, East Himalaya, Hainan, Jawa, Laos, Malaysia, Maluku, Myanmar, } \\
\text { New Guinea, Nicobar Is., Philippines, Santa Cruz Is., Solomon Is., Sumatera, Thailand, } \\
\text { Vanuatu, Vietnam }\end{array}$ & Gen, Ban \\
\hline Chrysoglossum ornatum Blume & $\mathrm{T}$ & - & II & - & $\begin{array}{l}\text { Assam, Borneo, Cambodia, China South-Central, China Southeast, East Himalaya, Fiji, Hainan, } \\
\text { India, Jawa, Laos, Malaysia, Myanmar, Nepal, New Caledonia, New Guinea, Philippines, } \\
\text { Samoa, Sri Lanka, Sulawesi, Sumatera, Taiwan, Thailand, Tibet, Vanuatu, Vietnam }\end{array}$ & Gen, Pas, Gon \\
\hline Cleisostoma discolor Lindl. & $\mathrm{E}$ & - & II & - & $\begin{array}{l}\text { Assam, Borneo, Cambodia, East Himalaya, India, Jawa, Laos, Lesser Sunda Is., Malaysia, } \\
\text { Sumatera, Thailand, Vietnam }\end{array}$ & Ban \\
\hline $\begin{array}{l}\text { Coelogyne miniata (Blume) } \\
\text { Lindl.** }\end{array}$ & $\mathrm{E}$ & - & II & - & Jawa, Lesser Sunda Is., Sumatera & Gaj, Maw, Pun \\
\hline $\begin{array}{l}\text { Coelogyne speciosa (Blume) } \\
\text { Lindl.** }\end{array}$ & $\mathrm{E}$ & - & II & - & Jawa, Lesser Sunda Is., Sumatera & $\begin{array}{l}\text { Gen, Kal, Pas, } \\
\text { Gon, Ban, Gaj, } \\
\text { Wat, Maw, Ind }\end{array}$ \\
\hline $\begin{array}{l}\text { Corymborkis veratrifolia (Reinw.) } \\
\text { Blume }\end{array}$ & $\mathrm{T}$ & - & II & - & $\begin{array}{l}\text { Andaman Is., Assam, Bangladesh, Bismarck Archipelago, Borneo, Cambodia, Caroline Is., } \\
\text { China South-Central, China Southeast, Christmas I., East Himalaya, Fiji, India, Jawa, Laos, } \\
\text { Lesser Sunda Is., Malaysia, Maluku, Marianas, Myanmar, Nansei-shoto, New Guinea, Nicobar } \\
\text { Is., Ogasawara-shoto, Philippines, Queensland, Samoa, Santa Cruz Is., Society Is., Solomon Is., } \\
\text { Sri Lanka, Sulawesi, Sumatera, Taiwan, Thailand, Tonga, Vanuatu, Vietnam, Wallis-Futuna Is. }\end{array}$ & Gen, Ban, Gond \\
\hline $\begin{array}{l}\text { Crepidium acuminatum (D.Don) } \\
\text { Szlach. }\end{array}$ & $\mathrm{T}$ & - & II & - & $\begin{array}{l}\text { Andaman Is., Assam, Bangladesh, Cambodia, China South-Central, China Southeast, East } \\
\text { Himalaya, India, Jawa, Laos, Myanmar, Nepal, Nicobar Is., Northern Territory, Philippines, } \\
\text { Sumatera, Thailand, Tibet, Vietnam, West Himalaya }\end{array}$ & Gen \\
\hline $\begin{array}{l}\text { Crepidium junghuhnii (J.J.Sm.) } \\
\text { Szlach.* }\end{array}$ & $\mathrm{E}$ & - & II & - & Jawa & Gen, Gaj \\
\hline $\begin{array}{l}\text { Crepidium kobi (J.J.Sm.) M.A.Clem. } \\
\text { \& D.L.Jones* }\end{array}$ & $\mathrm{T}$ & - & II & - & Jawa & Gen, Maw \\
\hline Crepidium perakense (Ridl.) Szlach. & $\mathrm{T}$ & - & II & - & Borneo, Jawa, Malaysia, Thailand & Ban \\
\hline Crepidium sp. & $\mathrm{T}$ & - & II & - & N/A & Gaj, Maw \\
\hline
\end{tabular}




\begin{tabular}{|c|c|c|c|c|c|c|}
\hline $\begin{array}{l}\text { Cymbidium bicolor subsp. } \\
\text { pubescens (Lindl.) Du Puy \& } \\
\text { P.J.Cribb }\end{array}$ & $\mathrm{E}$ & - & II & 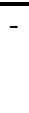 & $\begin{array}{l}\text { Andaman Is., Borneo, Cambodia, Jawa, Lesser Sunda Is., Malaysia, Nicobar Is., Philippines, } \\
\text { Sulawesi, Sumatera }\end{array}$ & Gen \\
\hline Cymbidium lancifolium Hook. & $\mathrm{T}$ & - & II & - & $\begin{array}{l}\text { Assam, Bangladesh, Borneo, Cambodia, China South-Central, China Southeast, East Himalaya, } \\
\text { Hainan, Japan, Jawa, Korea, Laos, Malaysia, Maluku, Myanmar, Nansei-shoto, Nepal, New } \\
\text { Guinea, Sulawesi, Sumatera, Taiwan, Thailand, Tibet, Vietnam, West Himalaya }\end{array}$ & Gen, Ban \\
\hline Dendrobium atavus J.J.Sm.* & $\mathrm{E}$ & - & II & - & Jawa & Ban \\
\hline Dendrobium aureilobum J.J.Sm. & E & - & II & - & Jawa, Malaysia, Sumatera & Ban, Wat \\
\hline Dendrobium barbatum Breda & $\mathrm{E}$ & - & II & - & Bangladesh, Jawa, Sumatera & $\begin{array}{l}\text { Gen, Kal, Pas, } \\
\text { Gon, Ban, Wat }\end{array}$ \\
\hline $\begin{array}{l}\text { Dendrobium connatum (Blume) } \\
\text { Lindl. }\end{array}$ & $\mathrm{E}$ & - & II & - & Borneo, Jawa, Lesser Sunda Is., Malaysia, Maluku, Sumatera, Thailand & Gen \\
\hline Dendrobium conspicuum Bakh.f. & $\mathrm{E}$ & - & II & - & China South-Central, Jawa, Lesser Sunda Is., Sumatera, Vietnam & Gaj \\
\hline Dendrobium crumenatum $\mathrm{Sw}$. & $\mathrm{E}$ & - & II & - & $\begin{array}{l}\text { Andaman Is., Borneo, Cambodia, Christmas I., India, Jawa, Laos, Lesser Sunda Is., Malaysia, } \\
\text { Maluku, Myanmar, New Guinea, Nicobar Is., Philippines, Sri Lanka, Sulawesi, Sumatera, } \\
\text { Taiwan, Thailand, Vietnam }\end{array}$ & $\begin{array}{l}\text { Gen, Kal, Pas, } \\
\text { Gon, Ban, Wat }\end{array}$ \\
\hline $\begin{array}{l}\text { Dendrobium lobulatum Rolfe ex } \\
\text { J.J.Sm.** }\end{array}$ & $\mathrm{E}$ & - & II & - & Borneo, Jawa, Maluku, Sumatera & $\begin{array}{l}\text { Gen, Kal, Pas, } \\
\text { Gon, Ban, Gaj, } \\
\text { Wat }\end{array}$ \\
\hline Dendrobium macrostachyum Lindl. & $\mathrm{E}$ & - & II & - & $\begin{array}{l}\text { Andaman Is., Bangladesh, Borneo, India, Jawa, Lesser Sunda Is., Malaysia, Maldives, } \\
\text { Myanmar, Nepal, Nicobar Is., Queensland, Sri Lanka, Sulawesi, Sumatera, Thailand, Vietnam, } \\
\text { West Himalaya }\end{array}$ & Ban \\
\hline $\begin{array}{l}\text { Dendrobium mutabile (Blume) } \\
\text { Lindl.** }\end{array}$ & $\mathrm{E}$ & - & II & - & Jawa, Lesser Sunda Is., Sumatera & $\begin{array}{l}\text { Gen, Kal, Pas, } \\
\text { Gon, Ban, Gaj, } \\
\text { Wat, Maw, Ind }\end{array}$ \\
\hline $\begin{array}{l}\text { Dendrobium nudum (Blume) } \\
\text { Lindl.** }\end{array}$ & $\mathrm{E}$ & - & II & - & Borneo, Jawa, Sumatera & Gaj \\
\hline Dendrobium sagittatum J.J.Sm.** & $\mathrm{E}$ & - & II & - & Jawa, Lesser Sunda Is., Sumatera & $\begin{array}{l}\text { Gen, Kal, Pas, } \\
\text { Gon, Ban, Gaj, } \\
\text { Wat, Gond }\end{array}$ \\
\hline $\begin{array}{l}\text { Dendrobium salaccense (Blume) } \\
\text { Lindl. }\end{array}$ & $\mathrm{E}$ & - & II & - & $\begin{array}{l}\text { Andaman Is., Assam, Borneo, China South-Central, East Himalaya, Hainan, India, Jawa, Laos, } \\
\text { Lesser Sunda Is., Malaysia, Myanmar, Sri Lanka, Sumatera, Thailand, Tibet, Vietnam }\end{array}$ & Gaj, Gond \\
\hline $\begin{array}{l}\text { Dendrochilum aurantiacum } \\
\text { Blume*** }\end{array}$ & $\mathrm{E}$ & - & II & - & Jawa, Sumatera & Gaj \\
\hline $\begin{array}{l}\text { Dendrochilum pallidiflavens Blume } \\
\text { var. pallidiflavens }\end{array}$ & $\mathrm{E}$ & - & II & - & Borneo, Jawa, Lesser Sunda Is., Malaysia, Myanmar, Philippines, Sumatera, Thailand & Pas, Gond \\
\hline Epipogium roseum (D.Don) Lindl. & $\mathrm{T}$ & - & II & - & $\begin{array}{l}\text { Angola, Assam, Borneo, Cameroon, Central African Repu, China South-Central, China } \\
\text { Southeast, Congo, East Himalaya, Fiji, Ghana, Guinea, Gulf of Guinea Is., Hainan, India, Japan, } \\
\text { Jawa, Kenya, Laos, Lesser Sunda Is., Liberia, Malawi, Malaysia, Maluku, Myanmar, Nansei- } \\
\text { shoto, Nepal, New Caledonia, New Guinea, New South Wales, Nigeria, Pakistan, Philippines, } \\
\text { Queensland, Solomon Is., Sri Lanka, Sudan, Sulawesi, Sumatera, Taiwan, Thailand, Tibet, } \\
\text { Uganda, Vanuatu, Vietnam, West Himalaya, Zaïre } \\
\text { Jawa. Lesser Sunda Is. New Guinea }\end{array}$ & Gen, Pas \\
\hline
\end{tabular}




\begin{tabular}{|c|c|c|c|c|c|c|}
\hline Eria javanica $($ Sw.) Blume & $\mathrm{E}$ & - & II & - & $\begin{array}{l}\text { Assam, Bismarck Archipelago, Borneo, China South-Central, East Himalaya, Jawa, Laos, } \\
\text { Lesser Sunda Is., Malaysia, Maluku, Myanmar, New Guinea, Philippines, Sulawesi, Sumatera, } \\
\text { Taiwan, Thailand, Vietnam }\end{array}$ & Kal \\
\hline Erythrodes blumei (Lindl.) Schltr. & $\mathrm{T}$ & $\mathrm{LC}$ & II & - & Jawa, Malaysia, Nicobar Is., Sumatera & Gen, Kal \\
\hline Eulophia zollingeri (Rchb.f.) J.J.Sm. & $\mathrm{T}$ & - & II & - & $\begin{array}{l}\text { Andaman Is., Assam, Borneo, China South-Central, China Southeast, East Himalaya, India, } \\
\text { Japan, Jawa, Malaysia, Maluku, Myanmar, Nansei-shoto, New Guinea, Ogasawara-shoto, } \\
\text { Philippines, Queensland, Sri Lanka, Sulawesi, Sumatera, Taiwan, Thailand, Vietnam }\end{array}$ & Gen \\
\hline Gastrochilus sororius Schltr. & $\mathrm{E}$ & - & II & - & Borneo, Java, Phillipines, Sulawesi, Sumatera & Gond \\
\hline Goodyera bifida (Blume) Blume & $\mathrm{T}$ & - & II & - & Borneo, Jawa, Lesser Sunda Is., Malaysia, Sulawesi, Sumatera, Thailand & Maw \\
\hline $\begin{array}{l}\text { Goodyera novembrilis (Rchb.f.) } \\
\text { Ormerod** }\end{array}$ & $\mathrm{T}$ & - & II & - & Jawa, Lesser Sunda Is., Sumatera & Gen, Gon, Wat \\
\hline $\begin{array}{l}\text { Goodyera reticulata (Blume) } \\
\text { Blume* }\end{array}$ & $\mathrm{T}$ & - & II & - & Borneo, Jawa, Lesser Sunda Is., Vietnam & Gen, Pas \\
\hline Goodyera rubicunda (Blume) Lindl. & $\mathrm{T}$ & - & II & - & $\begin{array}{l}\text { Assam, Bismarck Archipelago, Borneo, China South-Central, East Himalaya, Fiji, Jawa, Lesser } \\
\text { Sunda Is., Malaysia, Maluku, Nansei-shoto, New Caledonia, New Guinea, Philippines, } \\
\text { Queensland, Samoa, Solomon Is., Sulawesi, Sumatera, Taiwan, Tonga, Vanuatu, Vietnam }\end{array}$ & Gen, Kal, Wat \\
\hline Habenaria loerzingii J.J.Sm.* & $\mathrm{T}$ & - & II & - & Jawa & Maw \\
\hline $\begin{array}{l}\text { Habenaria undulatifolia Frapp. ex } \\
\text { Cordem.* }\end{array}$ & $\mathrm{T}$ & - & II & - & Jawa & $\begin{array}{l}\text { Gen, Kal, Pas, } \\
\text { Ban, Wat }\end{array}$ \\
\hline Hetaeria lamellata Blume* & $\mathrm{T}$ & - & II & - & Jawa & Gen \\
\hline Liparis caespitosa (Lam.) Lindl. & $\mathrm{E}$ & - & II & - & $\begin{array}{l}\text { Assam, Bismarck Archipelago, Borneo, Cambodia, China South-Central, East Himalaya, Fiji, } \\
\text { Hainan, India, Jawa, Laos, Lesser Sunda Is., Madagascar, Malawi, Malaysia, Maluku, } \\
\text { Mauritius, Mozambique, Nepal, New Caledonia, New Guinea, Philippines, Réunion, Samoa, } \\
\text { Society Is., Solomon Is., Sri Lanka, Sulawesi, Sumatera, Tanzania, Thailand, Tibet, Tubuai Is., } \\
\text { Uganda, Vanuatu, Vietnam, West Himalaya }\end{array}$ & Wat, Gond \\
\hline Liparis condylobulbon Rchb.f. & $\mathrm{E}$ & - & II & - & $\begin{array}{l}\text { Borneo, Fiji, Jawa, Lesser Sunda Is., Maluku, Myanmar, New Caledonia, New Guinea, } \\
\text { Philippines, Queensland, Samoa, Santa Cruz Is., Solomon Is., Sulawesi, Sumatera, Taiwan, } \\
\text { Thailand, Vanuatu, Vietnam, Wallis-Futuna Is. }\end{array}$ & Gen, Pas \\
\hline Liparis elliptica Wight & $\mathrm{E}$ & - & II & - & $\begin{array}{l}\text { Assam, China South-Central, China Southeast, East Himalaya, Fiji, India, Japan, Jawa, Lesser } \\
\text { Sunda Is., Myanmar, Nansei-shoto, Nepal, New Caledonia, Philippines, Samoa, Sri Lanka, } \\
\text { Taiwan, Thailand, Tibet, Vietnam }\end{array}$ & Maw, Pun \\
\hline Liparis latifolia Lindl. & $\mathrm{E}$ & - & II & - & $\begin{array}{l}\text { Borneo, Hainan, Jawa, Lesser Sunda Island, Malaysia, Philippines, Sulawesi, Sumatera, } \\
\text { Thailand }\end{array}$ & Pas \\
\hline Liparis pallida (Blume) Lindl. & $\mathrm{E}$ & - & II & - & Jawa, Lesser Sunda Is., Philippines, Sumatera & Gen \\
\hline Liparis wightiana Thwaites & $\mathrm{E}$ & - & II & - & India, Jawa, Sri Lanka, Sumatera, Thailand & Gen \\
\hline Luisia javanica J.J.Sm.** & $\mathrm{E}$ & - & II & - & Jawa, Maluku, Sulawesi & $\begin{array}{l}\text { Gen, Kal, Pas, } \\
\text { Ban }\end{array}$ \\
\hline Luisia taurina J.J.Sm.** & $\mathrm{E}$ & - & II & - & Borneo, Jawa, Sumatera & Kal \\
\hline Macodes petola (Blume) Lindl. & $\mathrm{T}$ & - & II & - & Borneo, Jawa, Malaysia, Nansei-shoto, Philippines, Sumatera, Thailand & Gen \\
\hline Mycaranthes latifolia Blume & $\mathrm{E}$ & - & II & - & Borneo, Jawa, Lesser Sunda Is., Malaysia, Sulawesi, Sumatera, Thailand & $\begin{array}{l}\text { Gen, Kal, Pas, } \\
\text { Gon, Ban, Gaj, } \\
\text { Wat }\end{array}$ \\
\hline Mycaranthes oblitterata Blume & $\mathrm{E}$ & - & II & - & Borneo, Cambodia, Jawa, Lesser Sunda Is., Malaysia, Sumatera, Thailand, Vietnam & Gaj \\
\hline Nephelaphyllum pulchrum Blume & $\mathrm{T}$ & - & II & - & $\begin{array}{l}\text { China, Kambodia, Assam, East Himalaya, Laos, Myanmar, Thailand, Vietnam, Borneo, Java, } \\
\text { Malaysia, Phillipines, Sumatera }\end{array}$ & Gond \\
\hline
\end{tabular}


Phaius pauciflorus (Blume) Blume Phalaenopsis amabilis (L.) Blume

Pholidota articulata Lindl.

Pholidota carnea (Blume) Lindl.

Pholidota convallariae (E.C.Parish \& Rchb.f.) Hook.f.

Pholidota globosa (Blume) Lindl **

Pholidota imbricata Lindl.

\section{Phreatia plantaginifolia (J.Koenig)} Ormerod

Phreatia tjibodasana J.J.Sm.** Pinalia djaratensis Schltr.** Pinalia multiflora (Blume)

Kuntze**

Polystachya concreta (Jacq.) Garay \& H.R.Sweet

Andaman Is , Assam, Bangladesh, Bismarck Archipelago, Cambodia, Caroline Is., China South- Maw

Central, Cook Is., East Himalaya, Fiji, India, Jawa, Laos, Lesser Sunda Is., Malaysia, Maluku, Marianas, Myanmar, Nansei-shoto, Nepal, New Caledonia, New Guinea, Niue, Northern Territory, Pakistan, Philippines, Queensland, Samoa, Society Is., Solomon Is., Sulawesi, Sumatera, Taiwan, Thailand, Tibet, Tonga, Vanuatu, Vietnam, Wallis-Futuna Is., West Himalaya

Borneo, Cambodia, Fiji, India, Jawa, Malaysia, New Guinea, Nicobar Is., Sumatera, Thailand, Gen, Pas, Gon, Vietnam

Jawa, Lesser Sunda Is.

Borneo, Jawa, Lesser Sunda Is., Sumatera

Assam, Borneo, Cambodia, China South-Central, China Southeast, East Himalaya, Hainan, Gen, Gaj, Maw Japan, Jawa, Laos, Malaysia, Maluku, Myanmar, Nansei-shoto, Nepal, New Guinea, Philippines, Sulawesi, Sumatera, Taiwan, Thailand, Tibet, Vietnam

Borneo, Jawa, Lesser Sunda Is., Malaysia, Sumatera

Bismarck Archipelago, Borneo, Jawa, Lesser Sunda Is., Maluku, New Guinea, Philippines, Ind Queensland, Sulawesi, Sumatera

Assam, Borneo, Cambodia, China South-Central, East Himalaya, Jawa, Laos, Malaysia, Ban Myanmar, Nepal, Sulawesi, Sumatera, Thailand, Tibet, Vietnam, West Himalaya

Borneo, Jawa, Lesser Sunda Is., Malaysia, Maluku, New Guinea, Philippines, Sulawesi, Ban, Gond Sumatera, Thailand

Assam, China South-Central, East Himalaya, Jawa, Myanmar, Sumatera, Thailand, Tibet, Gaj, Pun Vietnam

Jawa, Lesser Sunda Is., Sumatera

Andaman Is., Assam, Bangladesh, Bismarck Archipelago, Borneo, Cambodia, China South- Gen, Kal, Pas,

Central, East Himalaya, Fiji, India, Jawa, Laos, Lesser Sunda Is., Malaysia, Maluku, Myanmar, Gon, Ban, Wat Nepal, New Caledonia, New Guinea, Nicobar Is., Philippines, Queensland, Santa Cruz Is., Solomon Is., Sri Lanka, Sulawesi, Sumatera, Thailand, Tibet, Tonga, Vanuatu, Vietnam, West Himalaya

Andaman Is., Borneo, Cambodia, Jawa, Lesser Sunda Is., Malaysia, Maluku, Philippines, Ban, Pun Sulawesi, Sumatera, Thailand, Vietnam

Jawa, Sumatera

Jawa, Sumatera

Jawa, Lesser Sunda Is., Sumatera

Andaman Is., Angola, Argentina Northwest, Bahamas, Bolivia, Borneo, Brazil North, Brazil Gen, Gon, Wat Northeast, Brazil South, Brazil Southeast, Brazil West-Central, Burundi, Cambodia, Cameroon, Cape Provinces, Cayman Is., Central African Repu, China South-Central, Colombia, Comoros, Congo, Cuba, Dominican Republic, Ecuador, Equatorial Guinea, Ethiopia, Florida, French Guiana, Gabon, Ghana, Guatemala, Guinea, Gulf of Guinea Is., Guyana, Haiti, India, Ivory Coast, Jamaica, Jawa, Kenya, KwaZulu-Natal, Laos, Leeward Is., Lesser Sunda Is., Liberia, Madagascar, Malawi, Malaysia, Maluku, Mauritius, Mexico Southeast, Mozambique, Nicobar Is., Nigeria, Northern Provinces, Panamá, Peru, Philippines, Puerto Rico, Rwanda, Réunion, Seychelles, Sierra Leone, Sri Lanka, Sudan, Sulawesi, Sumatera, Suriname, Swaziland, Tanzania, Thailand, Togo, Trinidad-Tobago, Uganda, Venezuela, Venezuelan Antilles, Vietnam, Windward Is., Zambia, Zaïre, Zimbabwe 


\begin{tabular}{|c|c|c|c|c|c|c|}
\hline $\begin{array}{l}\text { Pteroceras compressum (Blume) } \\
\text { Holttum }\end{array}$ & $\mathrm{E}$ & - & II & - & Jawa, Malaysia, Sumatera, Thailand & $\begin{array}{l}\text { Gen, Kal, Pas, } \\
\text { Gon, Ban, Wat }\end{array}$ \\
\hline Rhomboda cristata (Blume) Ormerod & $\mathrm{T}$ & - & II & - & Jawa, Lesser Sunda Is., Philippines & Gen \\
\hline $\begin{array}{l}\text { Rhomboda velutina (J.J.Sm.) } \\
\text { Ormerod** }\end{array}$ & $\mathrm{T}$ & - & II & - & Jawa, Lesser Sunda Is. & Gen \\
\hline Rhynchostylis retusa (L.) Blume & $\mathrm{E}$ & - & II & - & $\begin{array}{l}\text { Andaman Is., Assam, Bangladesh, Borneo, Cambodia, China South-Central, East Himalaya, India, } \\
\text { Jawa, Laos, Malaysia, Myanmar, Nepal, Nicobar Is., Philippines, Sri Lanka, Sumatera, Thailand, } \\
\text { Vietnam, West Himalaya }\end{array}$ & Ban, Gond \\
\hline $\begin{array}{l}\text { Schoenorchis juncifolia Reinw. ex } \\
\text { Blume }\end{array}$ & $\mathrm{E}$ & - & II & - & Borneo, Jawa, Lesser Sunda Is., Malaysia, Sumatera & Maw \\
\hline Spathoglottis aurea Lindl. & $\mathrm{T}$ & - & II & - & Borneo, Jawa, Lesser Sunda Is., Malaysia, New Guinea, Sulawesi, Sumatera, Thailand, Vietnam & Wat \\
\hline Spathoglottis plicata Blume & $\mathrm{T}$ & - & II & - & $\begin{array}{l}\text { Andaman Is., Assam, Bangladesh, Bismarck Archipelago, Borneo, Cambodia, Caroline Is., Cook Is., } \\
\text { East Himalaya, Fiji, India, Jawa, Laos, Lesser Sunda Is., Malaysia, Maluku, Marianas, Myanmar, } \\
\text { Nansei-shoto, New Caledonia, New Guinea, Nicobar Is., Niue, Philippines, Queensland, Samo, } \\
\text { Santa Cruz Is., Solomon Is., Sri Lanka, Sulawesi, Sumatera, Taiwan, Thailand, Tonga, Vanuatu, } \\
\text { Vietnam, Wallis-Futuna Is. }\end{array}$ & Maw \\
\hline Stereosandra javanica Blume & $\mathrm{T}$ & - & II & - & $\begin{array}{l}\text { Borneo, China South-Central, East Himalaya, Jawa, Malaysia a, Myanmar, Nansei-shoto, New } \\
\text { Guinea, Philippines, Samoa, Solomon Is., Sumatera, Taiwan, Thailand, Vietnam }\end{array}$ & Gen \\
\hline Taeniophyllum hasseltii Rchb.f. & $\mathrm{E}$ & - & II & - & Christmas I., Jawa, Malaysia, Thailand & Gen \\
\hline Tainia speciosa Blume & $\mathrm{T}$ & - & II & - & Borneo, Jawa, Malaysia, Sumatera, Thailand & Gen \\
\hline Thelymitra javanica Blume & $\mathrm{T}$ & - & II & - & Jawa, Philippines & Pun \\
\hline $\begin{array}{l}\text { Thrixspermum obtusum (Blume) } \\
\text { Rchb.f.* }\end{array}$ & $\mathrm{E}$ & - & II & - & Jawa & Maw \\
\hline Trichotosia pauciflora Blume & E & - & II & - & Borneo, Jawa, Lesser Sunda Is., Malaysia, Sumatera, Thailand & Gen, Pas \\
\hline Tropidia angulosa (Lindl.) Blume & $\mathrm{T}$ & - & II & - & $\begin{array}{l}\text { Andaman Is., Assam, Bangladesh, China South-Central, China Southeast, East Himalaya, India, } \\
\text { Jawa, Laos, Lesser Sunda Is., Malaysia, Myanmar, Philippines, Sumatera, Thailand, Tibet, Vietnam }\end{array}$ & Kal \\
\hline Tropidia curcugiloides Lindl. & $\mathrm{T}$ & - & II & - & $\begin{array}{l}\text { Andaman Is., Assam, Bangladesh, Borneo, Cambodia, China South-Central, China Southeast, East } \\
\text { Himalaya, Hainan, India, Jawa, Laos, Lesser Sunda Is., Malaysia, Myanmar, Sulawesi, Sumatera, } \\
\text { Taiwan, Thailand, Vietnam }\end{array}$ & Gen \\
\hline $\begin{array}{l}\text { Tuberolabium zollingeri (Rchb.f.) } \\
\text { Ormerod \& Juswara** }\end{array}$ & E & - & II & - & Jawa, Lesser Sunda Is. & $\begin{array}{l}\text { Gen, Pas, Ban, } \\
\text { Wat }\end{array}$ \\
\hline Vanda tricolor Lindl.** & E & - & II & - & Jawa, Lesser Sunda Is. & Gen, Ban, Gaj \\
\hline Zeuxine gracilis (Breda) Blume & $\mathrm{T}$ & - & II & - & Assam, Borneo, East Himalaya, India, Jawa, Malaysia, Sumatera & Gen \\
\hline
\end{tabular}

Pasigitan, Gon: Gonoharjo, Ban: Banyuwindu, Gaj: Gajah Mungkur, Wat: Watuondo, Gond: Gondang, Ind: Indrakila, Pun: Puncak, Maw: Mawar Camp 


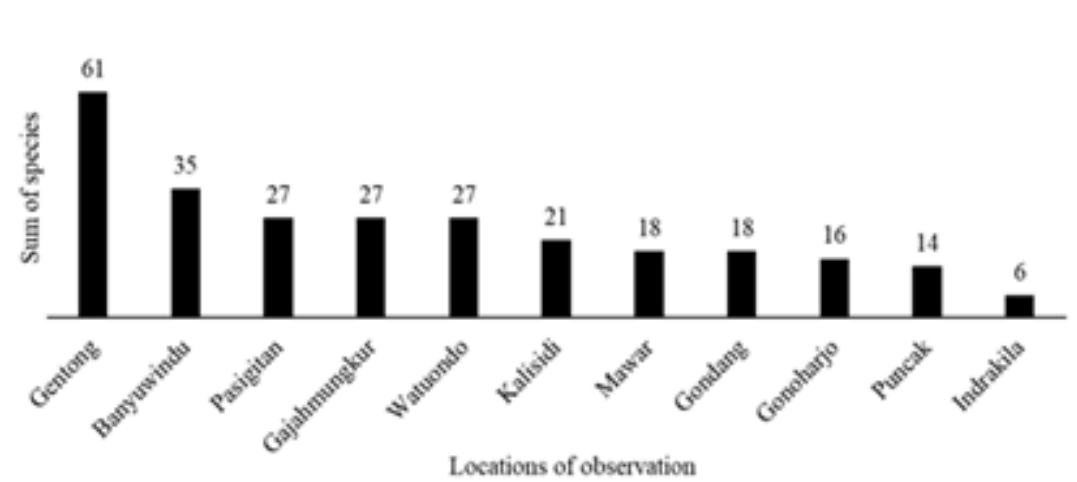

A

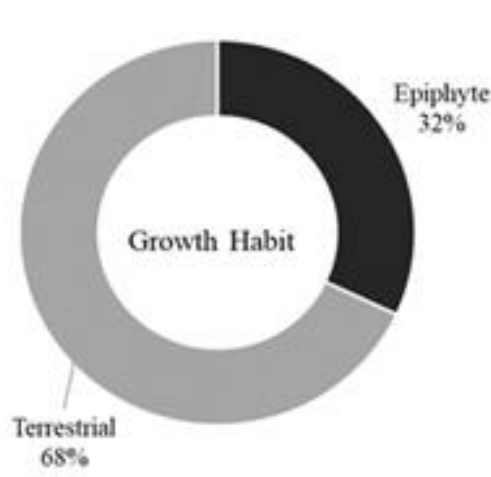

C

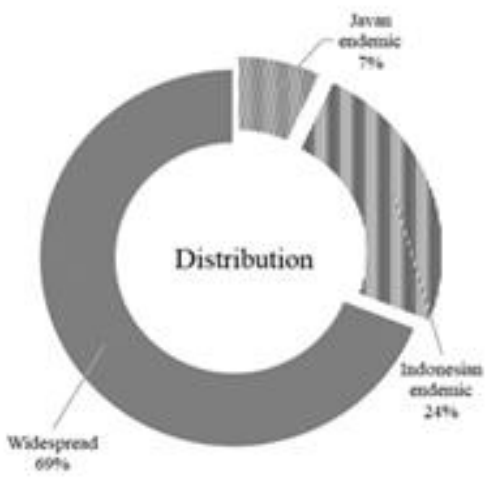

D

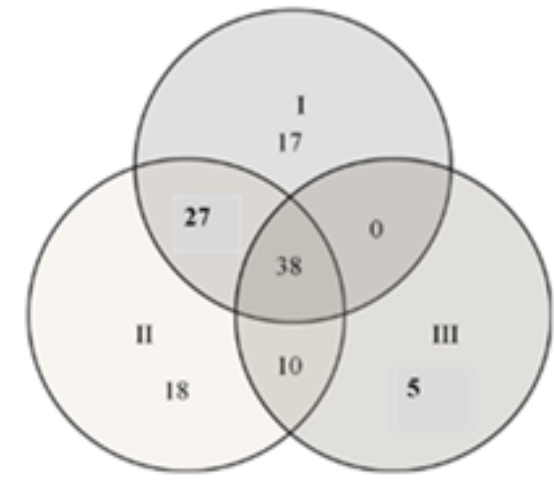

B

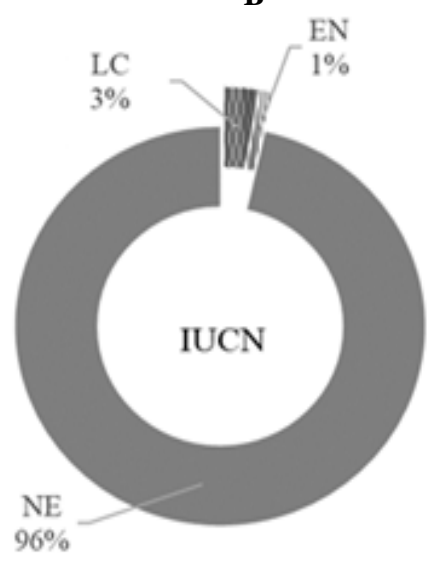

$\mathbf{E}$

Figure 2. The composition of orchids recorded in Mount Ungaran, Central Java, Indonesia. A. Location; B. Period of observation: I. 2010-2014, II. 2014-2018, and III. 2018-2021; C. Growth habits; D. Distribution; and E. Conservation status of IUCN

\section{Discussion}

Of the five sub-families of Orchidaceae, only three subfamilies occurred in Mount Ungaran, namely Cypripedioideae, Orchidoideae, and the rich one, Epidendroideae. Each sub-family had specific characteristics that can be used as a discriminant for grouping some genera with other related genera. The members of the Cypripedioideae sub-family are spectacular orchids with terrestrial on their life type and have distinct slipper-shaped pouches as the shape of their labellum. In this study, the sub-family was represented by only a single genus Paphiopedilum with a single species, namely $P$. javanicum. This endangered orchid has a green herbaceous leaf with many dark spots on the abaxial side. The main feature of Paphiopedilum is its pouch-like lip on the flower (Romadlon et al. 2021). While the diagnostic characteristics of $P$. javanicum are the presence of mottled leaves, with darker green spots superimposed over a light green background; the dorsal sepal is ovate, acute, green with white margins, longitudinally striped dark brown; petals long-oblong, held nearly horizontal but sometimes dropping, greenish finely spotted reddish-brown in the lower two-thirds, white or pink in the apical third; and the dull lip brownish-green (Comber 1990; Hendriyani et al. 2019)
The second sub-family in this study is Orchidoideae, a group of orchids that grow as terrestrial with erect inflorescences. Sub-family Orchidoideae shows several diagnostic characteristics such as their life form as terrestrial, the growing from tubers or a horizontal fleshy rhizome; anther basifixed or not; pollinia segmented, comprising massulae, or mealy (Chase et al. 2015). The Orchidoideae sub-family in this study was represented by 9 genera grouped into 3 tribes: Cranichideae, Diurideae, and Orchideae. The Cranichideae was represented by seven genera, including Anoectochilus, Erythrodes, Goodyera, Hetaeria, Rhomboda, Macodes, and Zeuxine. At the same time, the Diurideae tribe was represented by the genus Thelymitra, equal to Tribe Orchideae, which was only represented by the Habenaria genus.

As the largest sub-family in the Orchidaceae family ( $80 \%$ out of all orchid species), Epidendroideae has diverse characteristics (Dong et al. 2018). There are 26 known tribes as members of this sub-family (Freudenstein and Chase 2015). Although Epidendroideae has a diverse member with various variations, the members can be compiled into this group due to synthetic features such as a single anther that is entirely incumbent to the sub-erect. The anther form arises from column elongation or, as in the vandoids, from early anther bending. Most of these family members are epiphytes, but some tribes contain only 
terrestrial orchids. We found 45 genera in this sub-family which can be grouped into 11 tribes as follows: Tribe Arethuseae was represented by genera Arundina, Nephelaphyllum, Calanthe, Phaius, Spathoglottis, Tainia, and Ania; Tribe Collabieae was represented by genus Chrysoglossum; Tribe Coelogyneae was represented by genera Coelogyne, Pholidota, and Dendrochilum; Tribe Epidendreae was represented by genus Agrostophyllum; Tribe Epipogieae was represented by genera Epipogium and Stereosandra; Tribe Gastrodieae was represented by genus Nervilia; Tribe Malaxideae was represented by genera Crepidium and Liparis, Tribe Podochileae Bulbophyllum, Dendrobium, Eria, Bryobium, Mycaranthes, Pinalia, Trichotosia, Appendicula, Ceratostylis, and Phreatia; Tribe Tropidieae was represented by genera Tropidia and Corymborkis; Tribe Cymbidieae was represented by genera Acriopsis, Cymbidium, and Eulophia; Tribe Vandeae was represented by genera Aerides, Rhynchostylis, Phalaenopsis, Luisia, Bogoria, Cleisostoma, Thrixspermum, Vanda, Polystachya, Taeniophyllum, Schoenorchis, Gastrochillus, and Tuberolabium. Some studies showed that the average temperature on Mount Ungaran ranges from $22-27^{\circ} \mathrm{C}$ and the range of altitude is between 900-2,050 masl (Rahayuningsih et al. 2017). Such characteristics will support mosses as well as mycorrhizal fungi to life (Nurfadilah et al. 2016). Mosses and mycorrhizal fungi are important successors as natural orchids microhabitats (Kurniawan et al. 2020). The presence of this moss provides the moisture needed for orchid roots to grow and develop (Sathiyadash et al. 2012). In addition, the availability of large and old trees with many branches is also a factor in the number of orchid species, especially those with epiphytic life forms. Trees with these characteristics can store substrates that keep nutrients and water (Garcia et al. 2020; Kusumastuti et al. 2021). This condition was observed in Gentong (Rahayuningsih et al. 2015), so it was not surprising that the area had the most orchid species, and $50 \%$ were epiphytic species. That is also why the Epidendroideae subfamily, whose members are the most epiphytic species, had the most abundant wealth compared to other sub-families in Ungaran.

The species richness of Ungaran orchids is less than in some conservation areas in West Java. For example, in Gede-Pangrango National Park (NP) with 201 species. That is not surprising due to intensive care and more organized area by their zonation. However, the number of Mount Ungaran orchid species will not be much different compared to Java's eastern part conservation area with equal forestry management, such as Bromo Tengger Semeru NP with 135 species (Artaka 2019). Nevertheless, it means that Mount Ungaran has the potential for natural orchids' habitat.

Our previous study of orchids in Mount Ungaran only found 68 species (Utami et al. 2018). Another previous study of orchids in Ngesrepbalong village (Gentong site) showed 12 species (Dewi et al. 2018). The number of orchid species found in this study provides evidence that Mount Ungaran has a high richness of orchids and might still have an opportunity to record additional species. Interestingly, we also got field information from residents that other species from the type list compiled can still be found on the other side of Mount Ungaran.

The 2014-2018 observation recorded the highest number of orchid species, with 93 species, while the last observation period (2018-2021) found the lowest number of orchid species, with only 53 species. It is likely due to the forest fires in Mount Ungaran and the spread of COVID-19, which decreased the intensity and range of exploration during that period.

This study recorded the new distribution records of several species of orchids previously only found in West Java or East Java by Comber (1990). These species are Ania penangiana, Pholidota convallariae, and Crepidium junghuhnii. At the same time, some species are losing their endemic statuses, such as Goodyera reticulata, Bogoria raciborskii, and Taeniophyllum hasseltii (Averyanov et al. 2018; Ong et al. 2019; Hsu et al. 2020). In this study, we encountered several confusable and sympatric Crepidium species displaying the complex structure of the flowers. Based on the consultation with several experts, this species is a variation of $C$. koordersii. The description of the holotype that refers to $C$. koordersii is a diamond-shaped labellum, a tapered auricle in the shape of a triangle that narrows towards the base (Smith 1905; Comber 1990). However, the type we encountered was different, with the labellum tending to be rounded, the auricle kidney-shaped with rounded ends, and a purplish on both its tepals and sepals. The other discussion said this species is a natural hybrid between $C$. koordersii and $C$. Acuminatum. The Crepidium genus has pretty diverse members; at least 365 species have been known as members of this genus (Govaerts et al. 2021). Based on these reasons mentioned and the various member of the genus (Besi et al. 2020), it is essential to conduct a deeper study of the characteristics in more detail in live specimens and their herbarium so that we are in the process of waiting for the inflorescences for a more detailed study as a follow-up to the findings of this species and continue to designate this species as Crepidium sp.

Overexploitation of orchids to fulfill the orchid species market needs conservation action as soon as possible. Especially orchids with large and unique flowers, such as Bulbophyllum lobbii, Vanda tricolor, Aerides odorata, Rhynchostylis retusa, and $P$. javanicum. These orchids were rarely found in this study, and even the endangered $P$. javanicum was found only in one individual. In addition, Macodes petola, and Anoectochilus reinwardtii, which have unique leaves with glowing leaf veins, are the main attraction for orchid lovers. From the 11 observation locations, Gentong has the potential to be designated as an in-situ conservation area to conserve the remaining orchids of Mount Ungaran. Not only has a high level of species diversity, but forest cover on Gentong is also relatively good, with dense tree crowns that provide ideal habitat and microclimate to support orchid growth. Large trees and coffee plantations left without intensive care also become a suitable habitat for epiphytic orchids. 


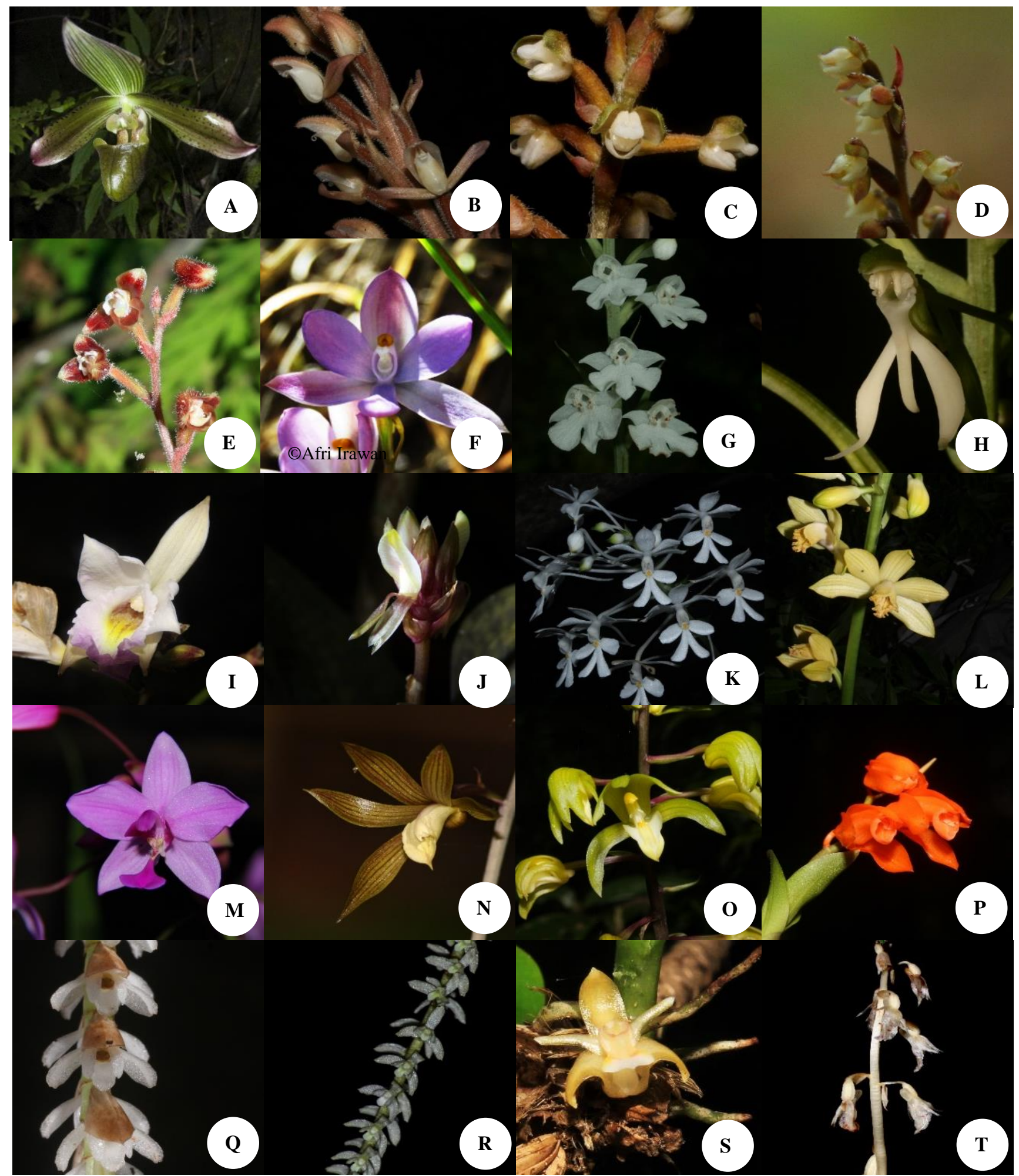

Figure 3. The representation of Mount Ungaran (Indonesia) Orchids is based on the sub-family: The representation of Ungaran Orchids in each sub-family. A. sub-family Cypripedioideae: Paphiopedilum javanicum. B-H. subfamily Orchidoideae: B. Goodyera, C. Hetaeria lamellata, D. Rhomboda cristata, E. Macodes petola, F. Erythrodes blumei, G. Habenaria loerzingii, H. H. undulata. I-T. Sub-family Epidendroideae: I. Arundina gramminifolia, J. Nephelaphyllum pulchrum, K. Calanthe triplicata, L. Phaius callosus, M. Spathoglottis plicata, N. Ania penangiana, O. Chrysoglossum ornatum, P. Coelogyne miniata, Q. Pholidota globosa, R. Dendrochilum pallideflavens; S. Agrostophyllum stipulatum subsp. bicuspidatum; T. Epipogium roseum 


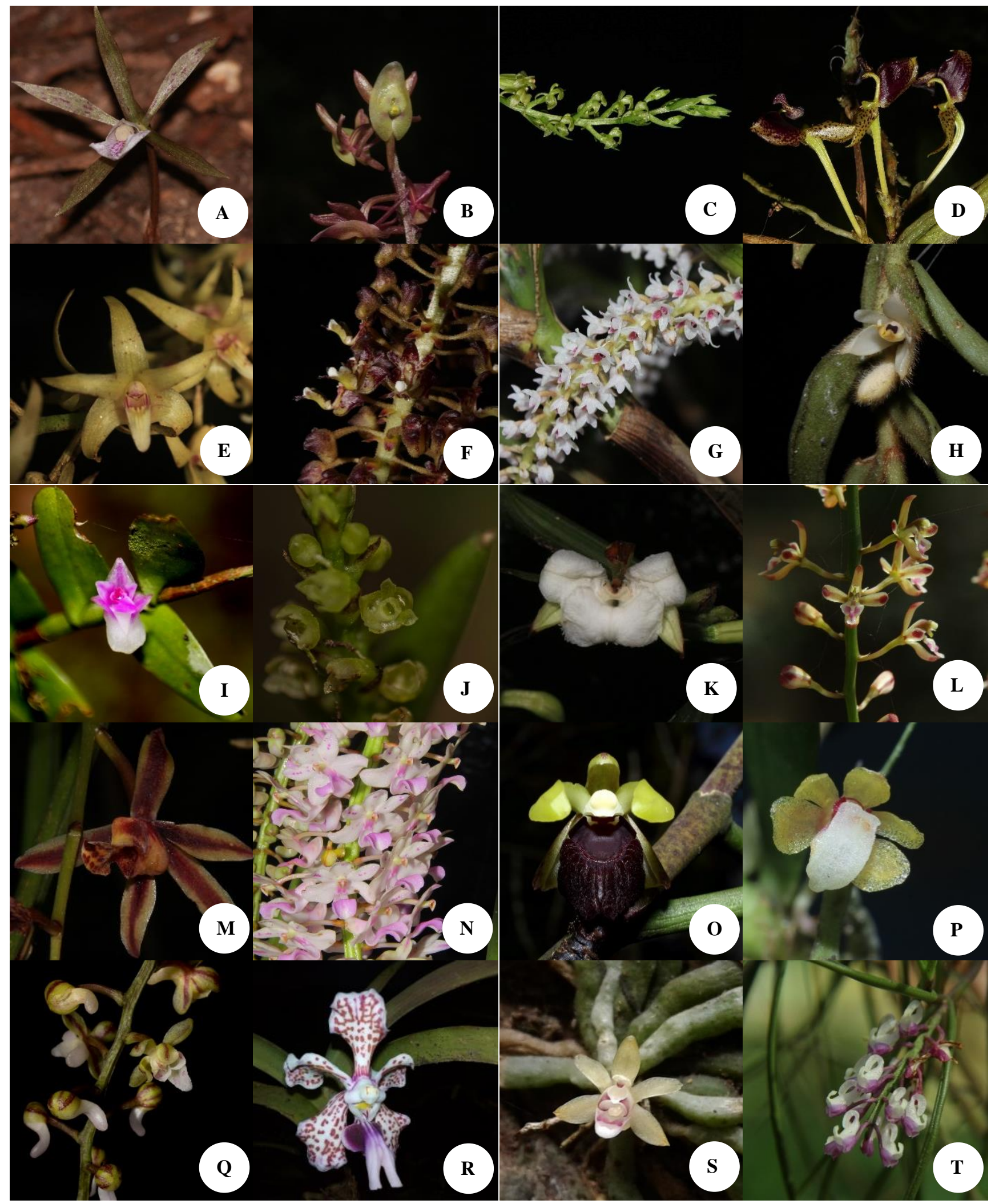

Figure 4. The representation sub-family Epidendroideae in Ungaran (continuation of figure 3): A. Nervilia punctata, B. Crepidium acuminatum, C. Liparis caespitsa, D. Bulbophyllum ecornutum subsp. ecornutum, E. Eria javanica, F. Mycaranthes latifolia, G. Pinalia multiflora, H. Trichotosia pauciflora, I. Appendicula ramosa, J. Phreatia plantaginifolia, K. Corymborkis veratrifolia, L. Acriopsis liliifolia var. liliifolia, M. Cymbidium bicolor subsp. pubescens, N. Rhynchostylis retusa, O. Luisia taurina, P. Bogoria raciborskii, Q. Cleisostoma discolor, R. Vanda tricolor, S. Taeniophyllum hasseltii, T. Schoenorchis juncifolia 

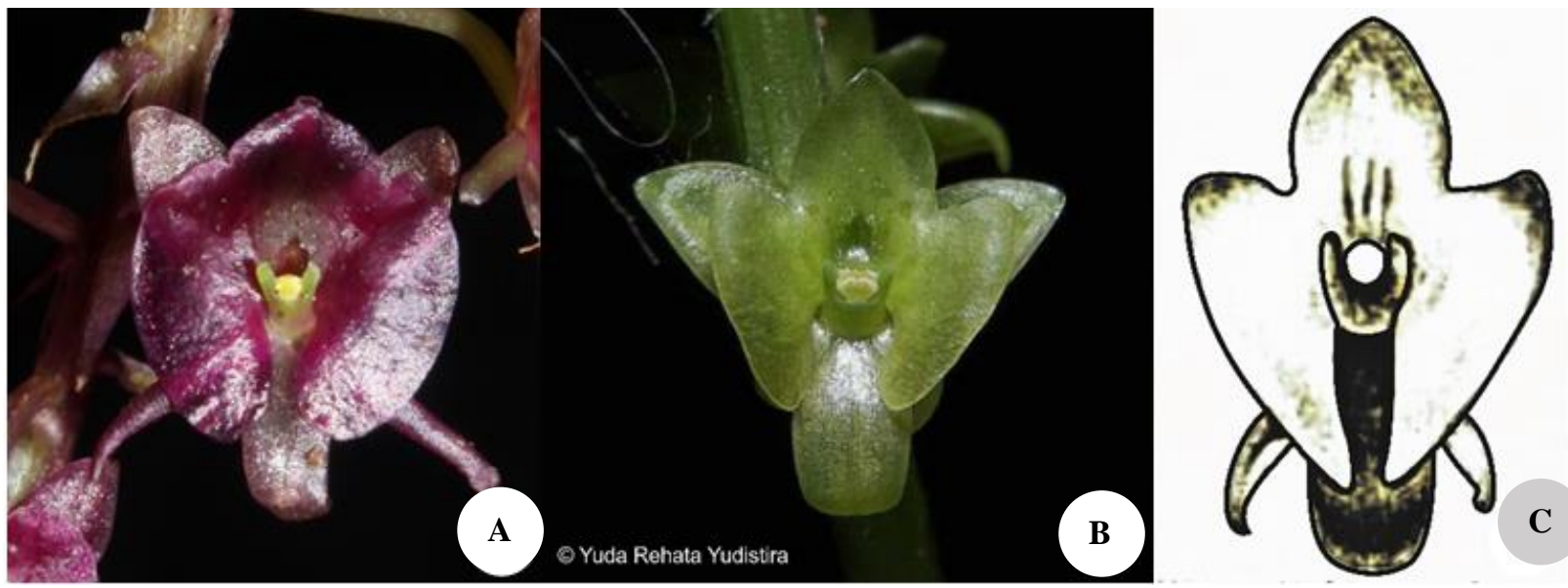

Figure 5. Crepidium sp found in this research (A), compared with C.koordersii from the other side in west Java (B) and digitized sketch from Smith (1908) (C). Photo of C.koordersii by Yuda Rehata Yudistira

In conclusion, 115 orchid species were found in Mount Ungaran, highlighting that this area serves as a critical habitat for wild orchids. Among all species recorded, at least 27 species were known to be endemic to Indonesia, and 8 species were endemic to the island of Java, of which four species were listed in the IUCN Red List. Besides that, all of the documented species were listed in the Appendix II category of the CITES, except one species, $P$. javanicum, which is listed in Appendix I. Gentong is recommended as a priority for an in-situ orchid protection area due to the highest number of species recorded which contributed to $54 \%$ of all orchids found. Yet, future exploration efforts are still needed in the other area of Mount Ungaran to obtain complete information on orchid diversity to support its conservation and enrich the knowledge of orchids in the region.

\section{ACKNOWLEDGEMENTS}

We would like to thank Perhutani, who gave the research permission. Muhammad Abdullah and Rahayu Nur Utami from the Department of Biology, Unnes, provide financial support in the second and third observation periods. Suparmin from Kendal served consumption and accommodation during the study. All of the Mensen van Biology from the ecology laboratory, Green Community, Pelatuk Bird Study Club, Universitas Negeri Semarang (Unnes), especially Novian Fitri, Nur Maulidatul Khasanah, Fathurrahman Siddiq, Solichin Danish Anggarani, Bayu Dwi Hadmoko, Ifan Syahputra, Kurnia Ramadhan, and Ahmad Navi Addawwami for gathering during survey periods. Yuda Rehata Yudistira and Afri Irawan for the beautiful pictures of Crepidium koordersii and Thelymitra javanica. Toni Artaka from Bromo Tengger Semeru National Park, W. A. Mustaqin from Genbi Foundation, Sulistiyono from Sanatadharma University, and Gilang Dwi Nugroho from UNS as experts who validated the scientific identity of several species.

\section{REFERENCES}

Agustini V, Zebua LI, Wenda N. 2016. Short Communication : Inventory of native orchids in Makki Sub-District, Lanny Jaya, Papua, Indonesia. Biodiversitas $17 \quad$ (1): 301-305. DOI: 10.13057/biodiv/d170141.

Artaka T. 2019. Anggrek Taman Nasional Bromo Tengger Semeru. Balai Besar Taman Nasional Bromo Tengger Semeru, Malang. [Indonesian]

Asseleih LMC, Garcia RAM, Cruz JYSR. 2015. Ethnobotany, pharmacology and chemistry of medicinal orchids from Veracruz. J Agric Sci Technol 5 (1): 745-754. DOI: 10.17265/21616256/2015.09.006.

Attri LK, Kant R. 2011. Orchid pollination: An observation on pollination-pollinator interaction in Cymbidium pendulum (Sw.) Roxb. Curr Bot 2 (7): 5-8.

Averyanov LV, Nguyen VC, Truong BV, Maisak TV, Luu HT, Nguyen KS, Dinh QD, Nguyen HT, Chu XC, Tran G, Nguyen VK, Le HS. 2018. New orchids (Orchidaceae: Cymbidieae and Vandeae) in the flora of Vietnam. Taiwania 63 (2): 119-138. DOI: 10.6165/tai.2018.63.119

Besi EE, Nikong D, Mustafa M, Go R. 2019. New records for orchids in Terengganu and Kelantan, Malaysia. J Sustain Sci Manag 2: 1-25.

Besi EE, Nikong D, Mustafa M, Seok C, Yong Y, Go R. 2020. Taxonomic placement of four confusable Crepidium species (Orchidaceae, Malaxidinae) based on macro-and micromorphological analyses, including a note on two new records to Peninsular Malaysia. Phytotaxa 454 (1): 31-44. DOI: 10.11646/phytotaxa.454.1.3.

Birdlife International. 2021. Data Zone Important Bird Area. http://datazone.birdlife.org/site/mapsearch

Biswas S, Singh DR. 2019. A Manual on Orchid Education (Issue August). ICAR-National Research Center for Orchids, East Sikkim, India

Chase MW, Cameron KM, Freudenstein JV, Pridgeon AM, Salazar G, Berg CVD, Schuiteman A. 2015. An updated classification of Orchidaceae. Bot $\mathrm{J}$ of the Linn Soc 177: 151-174. DOI: 10.1111/boj.12234.

Comber JB. 1990. Orchids of Java. The Bentham-Moxon Trust, London.

De L. 2020. Morphological diversity in orchids. Intl J Bot Stud 5 (5): 299238. DOI: 10.13140/RG.2.2.24041.31849.

Dewi ERS, Nugroho AS, Ulfa M. 2018. Menuju desa konservasi Anggrek di Desa Ngesrep Balong, Limbangan Kendal. J Dedicators Commun 2 (2): 100-107. DOI: 10.34001/jdc.v2i2.702. [Indonesian]

Dong WL, Wang RN, Zhang NY, Fan WB, Fang MF, Li ZH. 2018. Molecular evolution of chloroplast genomes of orchid species: Insights into phylogenetic relationship and adaptive evolution. Intl J Mol Sci 19 (3): 1-20. DOI: 10.3390/ijms19030716.

Freudenstein JV, Chase MW. 2015. Phylogenetic relationships in Epidendroideae (Orchidaceae), one of the great flowering plant 
radiations: Progressive specialization and diversification. Ann Bot 115: 665-681. DOI: 10.1093/aob/mcu253.

Garcia AM, Savelli EN, Canto SB, Mancera HA, Gauthier A, Cobos FA. 2020. Bark water storage capacity influences epiphytic orchid preference for host trees. Am J Bot 107 (5): 726-734. DOI: 10.1002/ajb.1470.

Govaerts R, Bernet P, Kratochvil K, Gerlach G, Carr G, Alrich P, Pridgeon AM, Pfahl J, Campacci MA, Baptista DH, Tigges H, Shaw J, Cribb P, George A, Kreuz K, Wood JJ. 2021. World Checklist of Orchidaceae. Kew Royal Botanic Gardens, London. Available from http://apps.kew.org/wcsp/

Hariyanto S, Pratiwi IA, Utami ESW. 2020. Seed morphometry of native indonesian orchids in the genus Dendrobium. Scientifica 2020 (1): 114. DOI: $10.1155 / 20203986369$.

Hendriyani E, Warseno T, Agnessya G, Oktavia E. 2019. Growth of slipper orchid Paphiopedilum javanicum (Reinw . ex Lindl .) Pfitzer during acclimatization stage. Biodjati 4(2): 291-297. DOI: 10.15575/biodjati.v4i2.5665.

Hsu TC, Chen CW, Hung HC, Tram NKT, Truong QC, Luu HT, Li CW. 2020. New and noteworthy orchids (Orchidaceae) discovered in Langbiang Plateau, Southern Vietnam. Taiwania 65 (2): 237-248. DOI: $10.6165 /$ tai.2020.65.237.

Kementerian Lingkungan Hidup (KLHK). 2018. Peraturan Menteri Lingkungan Hidup dan Kehutanan Republik Indonesia No P.106/MenLHK/Setjen/Kum.1/12/2018 tentang Perubahan Kedua atas Peraturan Menteri Lingkungan Hidup dan Kehutanan No. P.20/MenLHK/Setjen/Kum.1/6/2018 tentang Jenis Tumbuhan dan Satwa yang dilindungi. Kementerian Lingkungan Hidup, Jakarta. [Indonesian]

Kurniawan FY, Putri F, Suyoko A, Masyhuri H, Sulistianingrum MP, Semiarti E. 2020. The diversity of wild orchids in the southern slope of Mount Merapi, Yogyakarta, Indonesia eight years after the 2010 eruption. Biodiversitas 21 (9): 4457-4465. DOI: 10.13057/biodiv/d210964.

Kusumastuti NK, Suratman, Pitoyo A. 2021. Orchids diversity on six forest typesin Wasur National Park, Merauke, Papua, Indonesia.Asian J For 5: 101-110. DOI: 10.13057/asianjfor/r050206.

Nurfadilah S, Yulia ND, Ariyanti EE. 2016. Morphology, anatomy, and mycorrhizal fungi colonization in roots of epiphytic orchids of Sempu Island, East Java, Indonesia. Biodiversitas 17 (2): 592-603. DOI 10.13057/biodiv/d170229.
Ong PT, Tan JPT, Cacko RP. 2019. Clarification of the status of Teniophyllum hasseltii Rchb.f. in Peninsular Malaysia. Malay Orchid Rev 53: 85-89.

Pant B. 2013. Medicinal orchids and their uses: Tissue culture a potential alternative for conservation. Afr J Plant Sci 7 (10): 448-467. DOI: 10.5897/ajps2013.1031.

Rahayuningsih M, Kartijono NE, Suharini E. 2015. Spatial modeling of wreathed hornbill (Aceros undulatus) habitat in Mount Ungaran, Central Java. Intl J Environ Sci Dev 6 (6): 474-477. DOI: 10.7763/IJESD.2015.V6.640.

Rahayuningsih M, Kartijono N, Retnoningsih A. 2017. Short Communication: the nest characteristics of wreathed hornbill (Rhyticeros undulatus) in Mount Ungaran, Central Java, Indonesia. Biodiversitas 18 (3): 1130-1134. DOI: 10.13057/biodiv/d180334.

Rahayuningsih M, Kurniawan FH, Kartijono NE. 2020. The potential of Ficus species as frugivorous feed on gentong Hill, Mount Ungaran, Indonesia. For Ideas 26 (2): 540-548.

Rahayuningsih M, Utami NR, Tsabit AM, Abdullah M. 2017. Developing local wisdom to integrate etnobiology and biodiversity conservation in Mount Ungaran, Central Java Indonesia. Intl J Environ Ecol Eng 4 (9): 1-3. DOI: 10.1999/1307-6892/67613.

Romadlon MA, Zahra FA, Nugroho GD, Pitoyo A. 2021. Population, habitat characteristic, and modelling of endangered orchid, Paphiopedilum javanicum in Mount Lawu, Java, Indonesia. Biodiversitas 22 (4): 1996-2004. DOI: 10.13057/biodiv/d220448.

Sadili A, Sundari S. 2017. Diversity, distribution, and utilization of orchids species (Orchidaceae) in Bodogol Forest, Gede Pangrango National Park, West Java. Widyariset 3 (2): 95-106. DOI: 10.14203/widyariset.3.2.2017.95-106.

Sathiyadash K, Nadu T, Thangavelu M, Eswaranpillai U, Pandey RR. 2012. Mycorrhizal association and morphology in orchids. J Plant Interact 7 (3): 238-247. DOI: 10.1080/17429145.2012.699105.

Smith JJ. 1905. Die Orchideen von Java. In: Flora von Buiotenzorg. Leiden. DOI: 10.5962/bhl.title.86938.

Smith JJ. 1908. Die Orchideen von Java Figuren-Atlas. In: Flora von Buiotenzorg. Leiden.

Utami N, Rahayuningsih M, Abdullah M, Kurniawan F, Nazar L. 2018. 68 anggrek di Gunung Ungaran ( $1^{\text {st }}$ ed.). Unnes Press, Semarang. [Indonesian] 